\title{
Resposta à temperatura de Aphidius colemani Viereck (Hymenoptera, Braconidae, Aphidiinae) originário de três regiões climáticas de Minas Gerais, Brasil
}

\author{
Marcus Vinicius Sampaio', Vanda Helena Paes Bueno', Sandra Maria Morais Rodrigues ${ }^{2}$ \\ \& Maria da Conceição de Menezes Soglia ${ }^{1}$
}

${ }^{1}$ Universidade Federal de Lavras, Departamento de Entomologia, Caixa Postal 37, 37200-000 Lavras-MG. marcsampaio@yahoo.com.br, vhpbueno@ufla.br

${ }^{2}$ Embrapa-Algodão. Rua São Paulo, 790. Distrito Industrial, 78850-000 Primavera do Leste-MT. sandra@cnpa.embrapa.br

\begin{abstract}
Response to temperature of Aphidius colemani Viereck (Hymenoptera, Braconidae, Aphidiinae) from three climatic regions of Minas Gerais state, Brazil. The parasitoid Aphidius colemani Viereck, 1912 presents higher mortality at constant temperatures above $25^{\circ} \mathrm{C}$, and probably it is the cause of the failure on the biological control of Aphis gossypii Glover, 1877 when this parasitoid is used at higher temperatures in greenhouses. The aim of this work was to evaluate the influence of different temperatures on individuals of $A$. colemani from different climatic regions of Minas Gerais state, Brazil. Individuals of $A$. colemani were collected in the localities of Juramento, Lavras and São Gotardo, and reared in laboratory $\left(22 \pm 2^{\circ} \mathrm{C}, 70 \pm 10 \% \mathrm{RH}\right.$ and $12 \mathrm{~h}$ photophase) for three generations in the host A. gossypii, on cucumber plants. One mated female of $A$. colemani, $24-48 \mathrm{~h}$ old, was released for one hour, in a Petri dish $(15 \mathrm{~cm})$ with 20 nymphs of $2^{\text {nd }}$ instar of $A$. gossypii on a leaf disk of cucumber ( $4 \mathrm{~cm}$ of diameter) laid on a layer of agar-water $(1 \%)$. The parasitized aphids were kept at $16,19,22,25$ and $28 \pm 1{ }^{\circ} \mathrm{C}, 70 \pm 10 \% \mathrm{RH}$ and $12 \mathrm{~h}$ photophase. The emergence rates of individuals from Juramento (65.9 and 35.4\%) and São Gotardo (71.4 and 47.6\%) were lower compared to those from Lavras $\left(87.1\right.$ and $80.9 \%$ ) at 16 and $28^{\circ} \mathrm{C}$. The more suitable temperature for the development of $A$. colemani from Lavras was higher than those for individuals from Juramento and São Gotardo. The individuals from Lavras showed highest emergence at $28^{\circ} \mathrm{C}$, and this demonstrated the existence of individuals of $A$. colemani with tolerance to higher temperatures. These results open news perspectives on the use of different strains of A. colemani on biological control of A. gossypii in protected cultivation.
\end{abstract}

KeYwords. Aphis gossypii; biological control; biology; parasitoids; strains.

\begin{abstract}
Resumo. Resposta à temperatura de Aphidius colemani Viereck (Hymenoptera, Braconidae, Aphidiinae) originário de três regiões climáticas de Minas Gerais, Brasil. O parasitóide Aphidius colemani Viereck, 1912 apresenta alta mortalidade em temperaturas constantes superiores a $25^{\circ} \mathrm{C}$. Provavelmente esta é a causa do insucesso no controle biológico de Aphis gossypii Glover, 1877 com o uso de $A$. colemani em temperaturas elevadas em casas de vegetação. Este trabalho teve como objetivo avaliar a resposta a diferentes temperaturas de indivíduos de A. colemani, originários de diferentes regiões climáticas do estado de Minas Gerais. Indivíduos de A. colemani foram coletados nos municípios de Juramento, Lavras e São Gotardo, e criados em laboratório por três gerações em A. gossypii em plantas de pepino, em sala climatizada $\left(22 \pm 2^{\circ} \mathrm{C}, 70 \pm 10\right.$ UR e $12 \mathrm{~h}$ de fotofase). Uma fêmea de $A$. colemani, acasalada e com $24-48 \mathrm{~h}$ de vida, foi liberada por um período de uma hora em uma placa de Petri $(15 \mathrm{~cm})$ contendo 20 ninfas de $2^{\circ}$ ínstar de A. gossypii em um disco foliar de pepino (4 cm de diâmetro) sobre uma solução ágar/água a $1 \%$. Os pulgões parasitados foram mantidos em câmaras climáticas nas temperaturas de $16,19,22,25$ e $28 \pm 1^{\circ} \mathrm{C}$, com UR de $70 \pm 10 \%$ e fotofase de $12 \mathrm{~h}$. Nas temperaturas de 16 e $28^{\circ} \mathrm{C}$, a emergência dos parasitóides originários de Juramento $(65,9$ e 35,4\%) e São Gotardo $(71,4$ e $47,6 \%)$ foi significativamente inferior àquelas encontradas para os de Lavras $(87,1$ e $80,9 \%)$. A temperatura mais adequada para $o$ desenvolvimento de indivíduos de $A$. colemani oriundos de Lavras foi mais alta do que para aqueles oriundos de Juramento e São Gotardo. Indivíduos de Lavras apresentaram alta emergência a $28^{\circ} \mathrm{C}$, demonstrando a existência de indivíduos de $A$. colemani com tolerância a temperaturas mais altas. Esses resultados abrem novas perspectivas quanto a possibilidades de utilização de diferentes biótipos de A. colemani no controle biológico de A. gossypii em cultivos protegidos.
\end{abstract}

Palavras-Chave. Aphis gossypii; biologia; biótipos; controle biológico; parasitóides.

O pulgão Aphis gossypii Glover, 1877 (Hemíptera, Aphididae) é praga de importância agrícola em diversas partes do mundo (Blackman \& Eastop 1984), inclusive no Brasil (Bueno 1999). Dentre os principais fatores que dificultam o controle desta espécie pode-se citar o grande número de espécies hospedeiras (Blackman \& Eastop 1984), tolerância a temperaturas extremas, que vão desde $4^{\circ} \mathrm{C}$ (Bueno et al. 2004) até $30^{\circ} \mathrm{C}$ (Van Steenis \& El-Kawass 1995; Soglia et al. 2002), e a alta fecundidade (Van Steenis \& El-Kawass 1995; Soglia et al. 2003).
O parasitóide Aphidius colemani Viereck, 1912 (Hymenoptera, Braconidae, Aphidiinae) é um dos principais inimigos naturais de $A$. gossypii, apresentando grande potencial para ser utilizado no controle deste pulgão (Van Steenis 1993; Sampaio et al. 2001). Porém, falhas no controle de $A$. gossypii com a utilização de $A$. colemani vêm sendo relatadas no verão europeu em cultivos protegidos (Van Steenis \& El-Kawass 1995). Isso se deve, provavelmente, à alta mortalidade do parasitóide em temperaturas elevadas, já que $A$. colemani apresenta baixa emergência em temperaturas 
constantes superiores a $25^{\circ} \mathrm{C}$ (Toussidou et al. 1999; Sampaio 2004).

O sucesso do controle biológico de determinada praga, normalmente está relacionado com a tolerância à temperatura das populações da praga e do inimigo natural. Assim, é possível que, para o controle de uma determinada espécie de hospedeiro, sejam necessárias várias espécies de parasitóides ou de indivíduos de uma mesma espécie, porém, oriundos de populações adaptadas em diferentes condições climáticas (Messenger \& Van Den Bosch 1971). Indivíduos provenientes de populações de parasitóides originadas de regiões climaticamente diferentes podem apresentar desenvolvimento, fecundidade e mortalidade diferenciados, dependendo da temperatura à qual são expostos (Botto et al. 1988; Bleicher \& Parra 1989; Bleicher \& Parra 1990a b; Hopper et al. 1993; Mols \& Boers 2001; Royer et al. 2001; Liu et al. 2002).

No Brasil, somente o estado de Minas Gerais apresenta 10 regiões climáticas (Aspiazú et al. 1990). Desta forma, é grande a probabilidade de que populações de $A$. colemani, adaptadas em diferentes condições climáticas, encontrem-se distribuídas pelo país. Este trabalho teve como objetivo avaliar a resposta de indivíduos de $A$. colemani, originários de diferentes regiões climáticas do estado de Minas Gerais a diferentes temperaturas.

\section{MATERIAL E MÉTODOS}

Coleta dos parasitóides. Indivíduos do parasitóide $A$. colemani foram coletados em Juramento, Lavras e São Gotardo, municípios do Estado de Minas Gerais, os quais localizam-se em diferentes regiões climáticas. O município de Juramento (latitude $16^{\circ} 50^{\prime} 53^{\prime \prime}$ sul e longitude $43^{\circ} 35^{\prime} 13^{\prime \prime}$ oeste) apresenta clima Aw, caracterizado como tropical chuvoso, megatérmico com inverno seco e temperatura do mês mais frio superior a $18^{\circ} \mathrm{C}$; Lavras (latitude $21^{\circ} 14^{\prime} 43^{\prime \prime}$ sul e longitude $44^{\circ} 59^{\prime} 59^{\prime \prime}$ oeste), com clima $\mathrm{Cwb}$, caracterizado como temperado suave (mesotérmico), chuvoso com inverno seco, temperatura média do mês mais frio entre 3 e $18^{\circ} \mathrm{C}$ e do mês mais quente inferior a $22^{\circ} \mathrm{C}$; São Gotardo (latitude $19^{\circ} 18^{\prime} 40^{\prime \prime}$ sul e longitude $46^{\circ} 02^{\prime \prime} 56^{\prime \prime}$ oeste), com clima Cwa, caracterizado como temperado suave, que difere do clima de Lavras apenas pela temperatura média do mês mais quente superior a $22^{\circ} \mathrm{C}$ (Aspiazú et al. 1990).

Para a coleta dos parasitóides, partes de plantas com colônias de pulgões $A$. gossypii foram retiradas, levadas ao laboratório e acondicionadas em placas de Petri vedadas com tecido de organza até a formação das múmias, as quais foram individualizadas em tubos de vidro (10x8 mm) até a emergência dos parasitóides.

Dois casais de A. colemani/localidade foram obtidos sobre o pulgão hospedeiro Aphis spiraecola Patch, 1914 em Citrus sinensis (L.) (Rutaceae), nos municípios de Lavras e Juramento, e sobre Myzus persicae (Sulzer, 1776), em Solanum tuberosum L. (Solanaceae) em São Gotardo.

Criação de Aphis gossypii e Aphidius colemani. O pulgão A. gossypii foi mantido em plantas de pepino (Cucumis sativus
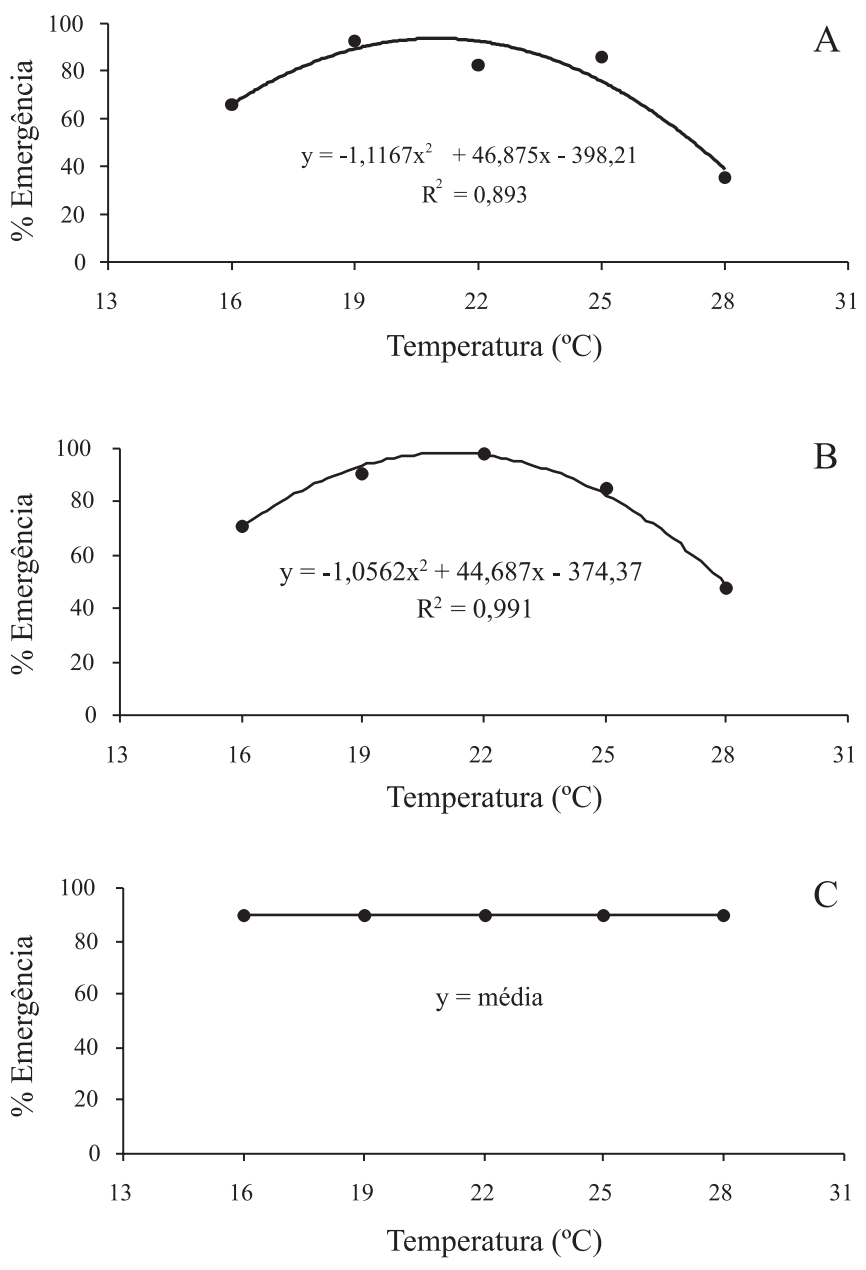

Fig. 1. Porcentagem de emergência de Aphidius colemani provenientes de três regiões climáticas de Minas Gerais em diferentes temperaturas, no hospedeiro Aphis gossypii. UR $70 \pm 10 \%$ e fotofase $12 \mathrm{~h}$. A, Juramento; B, São Gotardo; C, Lavras (MG).

L.) (Cucurbitaceae) em sala com temperatura ambiente. A criação de $A$. colemani foi realizada em sala climatizada $\left(22 \pm 2^{\circ} \mathrm{C}\right.$, UR de $70 \pm 10 \%$ e fotofase de $12 \mathrm{~h}$ ), utilizando-se uma gaiola de acrílico $(45 \times 90 \times 50 \mathrm{~cm})$ contendo um vaso com planta de pepino infestada com A. gossypii, sobre os quais os parasitóides, provenientes das diferentes regiões, foram criados por três gerações consecutivas antes de serem utilizados no experimento.

Resposta à temperatura de Aphidius colemani de diferentes regiões climáticas. Fêmeas de $A$. colemani, acasaladas e com 24-48h de vida, foram liberadas individualmente em uma placa de Petri $(15 \mathrm{~cm})$ contendo 20 ninfas de $2^{\circ}$ ínstar de $A$. gossypii em um disco foliar de pepino (4 $\mathrm{cm}$ de diâmetro) sobre uma camada de ágar/água a $1 \% \mathrm{e}$ mantida em câmara climatizada a $22 \pm 1^{\circ} \mathrm{C}$ por uma hora. Após este período, as fêmeas foram retiradas e os pulgões mantidos em câmaras climatizadas nas temperaturas de 16, 19, 22, 25 e $28 \pm 1^{\circ} \mathrm{C}$, com UR de $70 \pm 10 \%$ e fotofase de $12 \mathrm{~h}$ até a formação 
das múmias. Foram avaliados os pulgões parasitados por dez fêmeas do parasitóide de cada região para cada temperatura. Os pulgões foram observados diariamente e transferidos para novas placas contendo novo disco foliar, se necessário. Após a formação das múmias estas foram individualizadas em tubos de vidro (10x8 $\mathrm{mm}$ ) até a emergência dos adultos, os quais foram alimentados com mel puro logo após a emergência e com água diariamente, ambos depositados na forma de gotículas nas paredes do tubo.

Foram avaliados as porcentagens de parasitismo, de fêmeas e de emergência, o período de desenvolvimento e a longevidade dos parasitóides. As porcentagens foram calculadas agrupando-se os dados obtidos para cada fêmea de $A$. colemani avaliada $(\mathrm{n}=10)$. Para a avaliação do período de desenvolvimento foram utilizados todos os parasitóides emergidos e para a longevidade foram avaliados de 43 a 50 adultos de ambos os sexos para cada localidade em cada temperatura.

O experimento foi conduzido em delineamento inteiramente casualizado, em esquema fatorial $5 \times 3$ ( 5 temperaturas $\times 3$ regiões climáticas). Foi realizada análise da variância, e quando significativo, os dados foram submetidos à análise de regressão para avaliação do efeito da temperatura sobre indivíduos de $A$. colemani de cada região climática e teste de médias de Tukey, ao nível de significância de 5\%, para a comparação entre os indivíduos das diferentes regiões do parasitóide em cada temperatura.

O material - testemunha foi depositado no Museu Regional de Entomologia, do Departamento de Entomologia, da Universidade Federal de Lavras, em Lavras/MG.

\section{RESULTADOS}

Porcentagens de parasitismo, de fêmeas e de emergência de Aphidius colemani. Não foi constatada interação das temperaturas testadas com as diferentes regiões de origem do parasitóide (Tabela I). Entretanto, parasitóides de Lavras apresentaram parasitismo médio maior $(61,8 \%)$ em relação àqueles provenientes de São Gotardo $(48,7 \%)$, enquanto que para aqueles de Juramento, o parasitismo $(57,6 \%)$ não diferiu estatisticamente dos encontrados para as outras duas regiões (Tabela I). Para a porcentagem de fêmeas, indivíduos de São Gotardo $(65,5 \%)$ apresentaram média superior àquela encontrada para os de Juramento (49,4\%), e os parasitóides provenientes de Lavras $(60,5 \%)$ não apresentaram porcentagem média de fêmeas significativamente diferente daqueles das outras regiões (Tabela I).

Foi encontrada interação significativa para temperatura e origem dos parasitóides na porcentagem de emergência de $A$. colemani. Nas temperaturas de 16 e $28^{\circ} \mathrm{C}$, a emergência dos parasitóides de Juramento (65,9 e 35,4\%) e São Gotardo (71,4 e $47,6 \%)$ foi estatisticamente inferior àquelas encontradas para os de Lavras $(87,1$ e $80,9 \%)$. A $22^{\circ} \mathrm{C}$, indivíduos de Juramento apresentaram emergência média inferior à de São Gotardo, já para as demais temperaturas $\left(19\right.$ e $\left.25^{\circ} \mathrm{C}\right)$, não foi encontrada diferença significativa para $A$. colemani oriundos das três
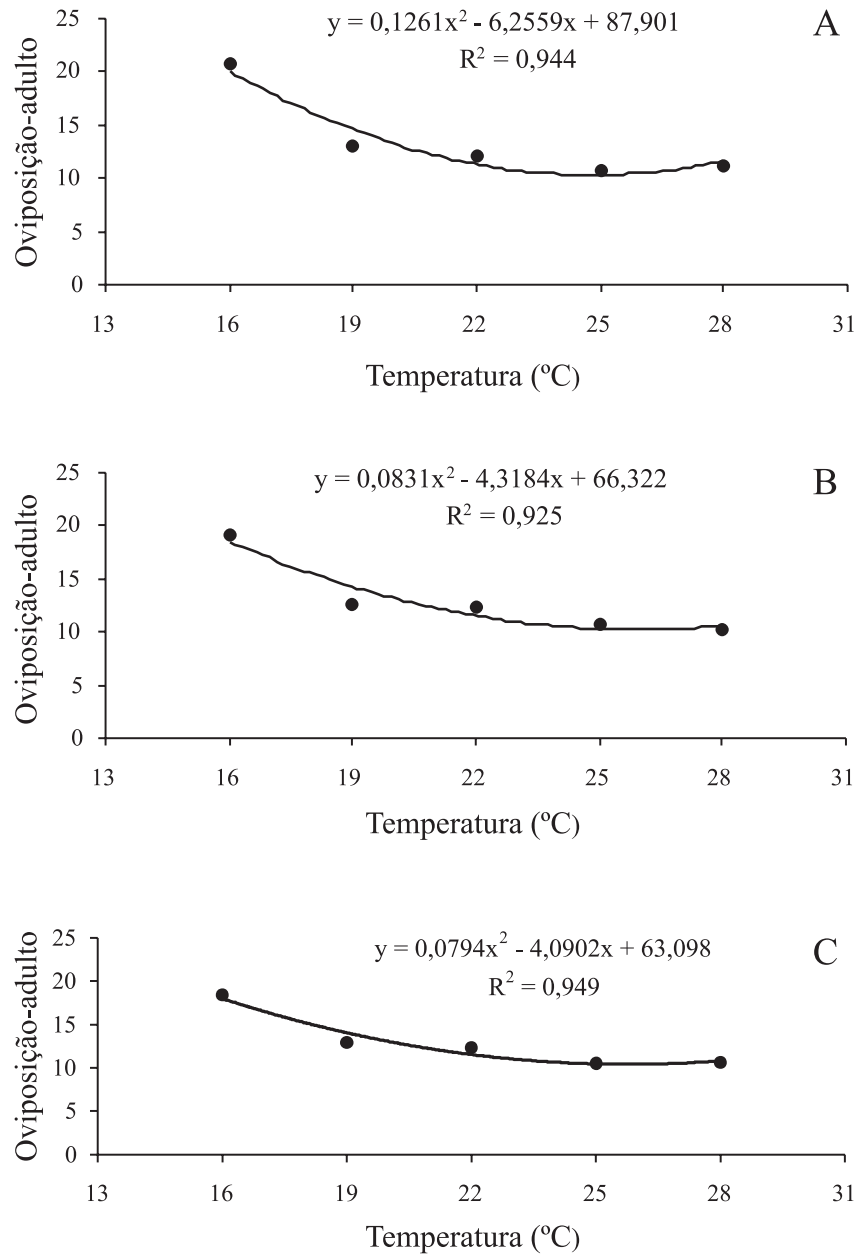

Fig. 2. Período de desenvolvimento (em dias) de Aphidius colemani provenientes de três regiões climáticas de Minas Gerais em diferentes temperaturas, no hospedeiro Aphis gossypii. UR $70 \pm 10 \%$ e fotofase 12h. A, Juramento; B, São Gotardo; C, Lavras (MG).

regiões climáticas (Tabela II). De modo geral, foi observado que os parasitóides provenientes de Juramento e São Gotardo apresentaram tendências semelhantes, com menor porcentagem de emergência nas temperaturas extremas (16 e $28^{\circ} \mathrm{C}$ ) e com pico de emergência próximo a $22^{\circ} \mathrm{C}$ (Figs. $1 \mathrm{~A}$ e 1B). Já a emergência dos parasitóides de Lavras não foi influenciada pela temperatura (Fig. 1C).

Período de desenvolvimento e longevidade. Houve interação significativa entre temperatura e origem de $A$. colemani para o período de desenvolvimento e a longevidade dos parasitóides. Com o aumento da temperatura de 16 para $28^{\circ} \mathrm{C}$, o desenvolvimento de $A$. colemani variou de 20,7 a 10,8; de 19,1 a 10,2 e de 18,4 a 10,5 dias para indivíduos de Juramento, São Gotardo e Lavras, respectivamente (Fig. 2 e Tabela III). Indivíduos de Juramento apresentaram período de desenvolvimento mais curto do que aqueles de Lavras e São Gotardo a $22^{\circ} \mathrm{C}(12,1$ dias). O período de desenvolvimento para os indivíduos de Lavras foi menor do que o encontrado 
para aqueles das demais regiões climáticas a $16^{\circ} \mathrm{C}(18,4$ dias $)$. Já para indivíduos de São Gotardo, o desenvolvimento foi de 12,7 e 10,2 dias a 19 e $28^{\circ} \mathrm{C}$ respectivamente, período este significativamente menor do que o encontrado para indivíduos de Juramento e Lavras nas mesmas temperaturas (Tabela III). A tendência observada para o período de desenvolvimento dos parasitóides provenientes das três regiões climáticas de Minas Gerais foi semelhante, ocorrendo uma diminuição do período de desenvolvimento com o aumento da temperatura no intervalo de 16 a $25^{\circ} \mathrm{C}$, e estabilização no intervalo de 25 a $28^{\circ} \mathrm{C}$ (Fig. 2).

A longevidade de A. colemani variou de 18,3 a 2,9 dias; 20,5 a 2,6 dias e de 23,5 a 2,9 dias, respectivamente para os indivíduos de Juramento, São Gotardo e Lavras, no intervalo de 16 a $28^{\circ} \mathrm{C}$ (Fig. 3 e Tabela III). Foi observado que a longevidade dos indivíduos provenientes de Juramento foi menor do que aqueles de Lavras nas temperaturas de 16,19 e $25^{\circ} \mathrm{C}$ e semelhantes a 22 e $28^{\circ} \mathrm{C}$ (Tabela III). Quando comparada à longevidade dos indivíduos de Juramento e São Gotardo, foi observado que indivíduos de Juramento apresentaram menor longevidade a 16,19 e $22^{\circ} \mathrm{C}$ e longevidade semelhante a 25 e $28^{\circ} \mathrm{C}$ (Tabela III). Os indivíduos de Lavras e São Gotardo apresentaram longevidades semelhantes nas temperaturas de $19,22,25$ e $28^{\circ} \mathrm{C}$, porém maior a $16^{\circ} \mathrm{C}$. Os parasitóides das três regiões climáticas apresentaram tendências semelhantes, diminuindo gradativamente a longevidade com o incremento da temperatura, porém, esta diminuição foi mais suave no intervalo de 19 a $25^{\circ} \mathrm{C}$ (Fig. 3).

\section{DISCUSSÃO}

Os resultados quanto ao parasitismo em A. gossypii encontrados no presente experimento, para os indivíduos de A. colemani das três regiões climáticas (entre 48,7 e 61,8\%), foram menores do que aqueles encontrados por Van Steenis (1993) (70 e 80\%) em temperaturas de 20 e $25^{\circ} \mathrm{C}$ em A. gossypii em cultivos de pepino em casas de vegetação na Holanda, e por Sampaio et al. (2001) $(75 \%)$ a $22^{\circ} \mathrm{C}$ em laboratório. Entretanto, pode ser considerado que a porcentagem de parasitismo de $A$. colemani foi alta para indivíduos das três regiões climáticas estudadas.

Ao comparar indivíduos das três regiões, verificou-se que para Lavras obteve-se maior porcentagem de parasitismo do que na de São Gotardo, podendo os parasitóides desta localidade apresentar maior eficiência no controle de $A$. gossypii. O mesmo pode ser inferido quanto a porcentagem de fêmeas, a qual foi maior para os indivíduos de São Gotardo do que para a de Juramento. Desta forma, indivíduos de São Gotardo podem apresentar vantagens no crescimento populacional em relação aqueles de Juramento.

A temperatura não afetou o parasitismo e a porcentagem de fêmeas, porém, exerceu grande influência na emergência de A. colemani originários de Juramento e São Gotardo, os quais apresentaram emergência reduzida nas temperaturas de $16 \mathrm{e}$ $28^{\circ} \mathrm{C}$. Toussidou et al. (1999) também encontraram queda da emergência de $A$. colemani a $28^{\circ} \mathrm{C}$. Já a emergência de
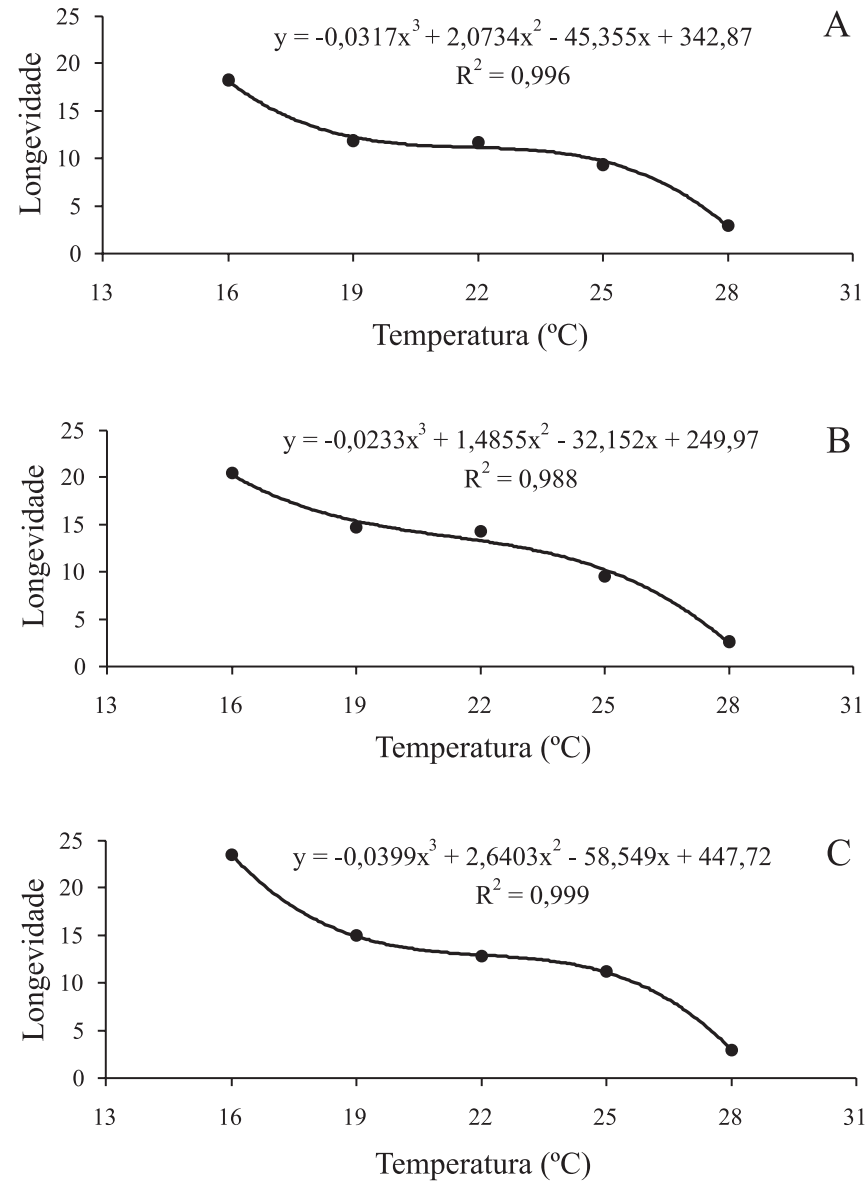

Fig. 3. Longevidade (em dias) de Aphidius colemani provenientes de três regiões climáticas de Minas Gerais em diferentes temperaturas, no hospedeiro Aphis gossypii. UR $70 \pm 10 \%$ e fotofase $12 \mathrm{~h}$. A,Juramento; B, São Gotardo; C, Lavras (MG)

indivíduos de Lavras não foi afetada pela temperatura, apresentando alta emergência nas cinco temperaturas avaliadas. A menor emergência verificada para os indivíduos de Juramento e São Gotardo a $16^{\circ} \mathrm{C}$, quando comparada aos de Lavras, provavelmente foi devida à adaptação do parasitóide à região de origem, já que Lavras é o local que apresenta as menores médias de temperatura (Aspiazú et al. 1990 e Ministério da Agricultura e Reforma Agrária, 1992). Royer et al. (2001) também encontraram maior mortalidade em temperaturas baixas para populações do parasitóide Lysiphlebus testaceipes (Cresson, 1880) (Hymenoptera, Aphidiidae) originadas de regiões mais quentes.

O período de desenvolvimento de A. colemani, embora influenciado pela temperatura e ter sido diferente entre os indivíduos das diferentes regiões climáticas, não indicou nenhum padrão que pudesse caracterizar os parasitóides quanto ao local de origem. Botto et al. (1988) verificou menor período de desenvolvimento para o parasitóide Aphidius ervi Haliday, 1834 provenientes de regiões mais quentes.

A longevidade de A. colemani foi influenciada 
Tabela I. Porcentagens de parasitismo e de fêmeas de Aphidius colemani provenientes de três regiões climáticas de Minas Gerais em diferentes temperaturas, no hospedeiro Aphis gossypii. UR $70 \pm 10 \%$ e fotofase $12 \mathrm{~h}$.

\begin{tabular}{ccccc}
\hline \multirow{2}{*}{ Temperatura $\left({ }^{\circ} \mathrm{C}\right)$} & \multicolumn{3}{c}{ Porcentagens médias } & \multirow{2}{*}{$\begin{array}{c}\text { Média } \\
\pm \text { erro padrão* }\end{array}$} \\
\cline { 2 - 4 } & Juramento & $\begin{array}{c}\text { Parasitismo }(\mathrm{n}=10) \\
\text { São Gotardo }\end{array}$ & Lavras & $59,8 \pm 4,22 \mathrm{~A}$ \\
16 & 61,2 & 56,4 & 61,8 & $49,2 \pm 4,63 \mathrm{~A}$ \\
19 & 46,8 & 44,4 & 56,6 & $55,0 \pm 3,31 \mathrm{~A}$ \\
22 & 56,9 & 47,4 & 60,6 & $56,4 \pm 5,08 \mathrm{~A}$ \\
25 & 55,1 & 50,2 & 63,8 & $59,6 \pm 5,24 \mathrm{~A}$ \\
\hline 28 & 67,8 & 45,0 & 66,1 & Média \\
\hline Média \pm erro padrão * & $57,6 \pm 3,90$ ab & $48,7 \pm 3,38 \mathrm{~b}$ & $61,8 \pm 3,04 \mathrm{a}$ & \pm erro padrão* \\
\hline \multirow{2}{*}{ Temperatura $\left({ }^{\circ} \mathrm{C}\right)$} & & Fêmeas & $55,8 \pm 4,79 \mathrm{~A}$ \\
& Juramento & São Gotardo & Lavras & $56,2 \pm 5,68 \mathrm{~A}$ \\
16 & $44,9(4) * *$ & $61,2(9)$ & $55,3(9)$ & $57,0 \pm 3,95 \mathrm{~A}$ \\
22 & $37,3(6)$ & $58,7(7)$ & $69,9(7)$ & $57,5 \pm 4,39 \mathrm{~A}$ \\
25 & $55,6(7)$ & $59,4(8)$ & $59,1(9)$ & $69,2 \pm 4,36 \mathrm{~A}$ \\
\hline
\end{tabular}

* Médias seguidas de mesma letra maiúscula nas colunas e minúscula nas linhas não diferem entre si pelo teste Tukey ( $\mathrm{p}<0,05)$.

** $\mathrm{n}=$ número de indivíduos avaliados.

significativamente pela temperatura e no geral, foi menor para os indivíduos de Juramento e semelhantes para aqueles de Lavras e São Gotardo na maioria das temperaturas, exceto a $16^{\circ} \mathrm{C}$, onde os indivíduos de Lavras apresentaram maior longevidade. Este fato também pode estar relacionado com a adaptação à região de origem do parasitóide.

No Brasil durante o programa de controle biológico dos pulgões do trigo, de 1978 a 1992, foram introduzidos indivíduos de A. colemani provenientes de populações da França e Israel (Salvadori \& Salles 2002), embora existam relatos da presença dessa espécie de parasitóide no país anteriormente a estas introduções (Silva et. al. 1968; Bartoszeck 1976; Pimenta \& Smith 1976). Este parasitóide apresenta alta mortalidade em temperaturas superiores a $25^{\circ} \mathrm{C}$ (Toussidou et al. 1999), temperaturas nas quais $A$. gossypii apresenta-se adaptado (Van Steenis \& El-Kawass 1995; Soglia et al. 2002; Soglia et al. 2003). Assim, para o sucesso no controle biológico de pragas que apresentam maior tolerância a temperaturas extremas que seus inimigos naturais, pode ser necessária a utilização de indivíduos de diferentes populações desses inimigos naturais, visando o controle em diferentes regiões climáticas. $\mathrm{Na}$ Califórnia (EUA), foram utilizados indivíduos de duas populações de Trioxys pallidus (Haliday, 1833) (Hymenoptera, Aphidiidae), adaptadas a diferentes condições climáticas, para o controle do pulgão Chromaphis juglandicola (Kaltenbach) em cultivos de nogueira no campo (Messenger \& Van Den Bosch 1971). De maneira análoga, o controle biológico de $A$. gossypii em diferentes épocas do ano, e em locais com grande variação de temperatura, pode ser incrementado pela utilização de diferentes biótipos de A. colemani. O entendimento se a espécie ou um biótipo do parasitóide pode sincronizar sua população com aquela do hospedeiro-alvo, em diferentes épocas do ano, é essencial para o sucesso no controle biológico de $A$. gossypii.

Tabela II. Porcentagem (média \pm erro padrão) de emergência de Aphidius colemani provenientes de três regiões climáticas de Minas Gerais em diferentes temperaturas, no hospedeiro Aphis gossypii. UR $70 \pm 10 \%$ e fotofase $12 \mathrm{~h}$.

\begin{tabular}{cccc}
\hline \multirow{2}{*}{ Temperatura $\left({ }^{\circ} \mathrm{C}\right)$} & \multicolumn{3}{c}{ Emergência de A. colemani } \\
\cline { 2 - 4 } & Juramento & São Gotardo & Lavras \\
\hline 16 & $65,9 \pm 4,01 \mathrm{~b}(9)^{* *}$ & $71,4 \pm 6,09 \mathrm{~b}(9)$ & $87,1 \pm 3,44 \mathrm{a}(10)$ \\
19 & $92,6 \pm 2,58 \mathrm{a}(8)$ & $90,9 \pm 5,02 \mathrm{a}(10)$ & $88,3 \pm 3,62 \mathrm{a}(9)$ \\
22 & $82,5 \pm 5,66 \mathrm{~b}(10)$ & $97,7 \pm 1,72 \mathrm{a}(10)$ & $95,4 \pm 1,63 \mathrm{ab}(10)$ \\
25 & $85,9 \pm 4,14 \mathrm{a}(8)$ & $85,0 \pm 4,59 \mathrm{a}(10)$ & $96,1 \pm 1,79 \mathrm{a}(10)$ \\
28 & $35,4 \pm 5,50 \mathrm{~b}(9)$ & $47,6 \pm 6,97 \mathrm{~b}(10)$ & $80,9 \pm 4,02 \mathrm{a}(9)$ \\
\hline
\end{tabular}

* Médias seguidas de mesma letra minúsculas nas linhas não diferem entre si pelo teste de Tukey $(\mathrm{p}<0,05)$.

** $\mathrm{n}=$ número de indivíduos avaliados 
Tabela III. Desenvolvimento (oviposição à emergência) e longevidade (em dias) de Aphidius colemani de três regiões climáticas de Minas Gerais em diferentes temperaturas, no hospedeiro Aphis gossypii. UR $70 \pm 10 \%$ e fotofase $12 \mathrm{~h}$.

\begin{tabular}{cccc}
\hline \multirow{2}{*}{ Temperatura $\left({ }^{\circ} \mathrm{C}\right)$} & \multicolumn{3}{c}{ Período (média \pm erro padrão) } \\
\cline { 2 - 4 } & Juramento & Desenvolvimento de A. colemani & Lavras \\
\hline 16 & $20,7 \pm 0,10 \mathrm{a}(79)^{* *}$ & São Gotardo & $18,4 \pm 0,07 \mathrm{c}(105)$ \\
19 & $13,0 \pm 0,04 \mathrm{a}(85)$ & $19,1 \pm 0,12 \mathrm{~b}(70)$ & $12,9 \pm 0,07 \mathrm{a}(97)$ \\
22 & $12,1 \pm 0,04 \mathrm{~b}(98)$ & $12,7 \pm 0,08 \mathrm{~b}(81)$ & $12,3 \pm 0,05 \mathrm{a}(112)$ \\
25 & $10,8 \pm 0,05 \mathrm{a}(94)$ & $12,4 \pm 0,08 \mathrm{a}(90)$ & $10,5 \pm 0,05 \mathrm{~b}(118)$ \\
28 & $11,2 \pm 0,09 \mathrm{a}(48)$ & $10,7 \pm 0,06 \mathrm{ab}(80)$ & $10,6 \pm 0,09 \mathrm{~b}(104)$ \\
\hline & & $10,2 \pm 0,07 \mathrm{c}(47)$ & Lavras \\
\hline Temperatura $\left({ }^{\circ} \mathrm{C}\right)$ & Luramento & Longevidade de A. colemani & $23,5 \pm 0,83 \mathrm{a}(50)$ \\
16 & $18,3 \pm 0,77 \mathrm{c}(50)^{* *}$ & São Gotardo & $15,0 \pm 0,44 \mathrm{a}(50)$ \\
19 & $11,9 \pm 0,50 \mathrm{~b}(50)$ & $20,5 \pm 0,88 \mathrm{~b}(50)$ & $12,8 \pm 0,53 \mathrm{ab}(50)$ \\
22 & $11,7 \pm 0,48 \mathrm{~b}(50)$ & $14,7 \pm 0,53 \mathrm{a}(50)$ & $11,2 \pm 0,28 \mathrm{a}(50)$ \\
25 & $9,4 \pm 0,40 \mathrm{~b}(50)$ & $14,3 \pm 0,53 \mathrm{a}(50)$ & $2,9 \pm 0,17 \mathrm{a}(50)$ \\
\hline
\end{tabular}

* Médias seguidas de mesma letra minúsculas nas linhas não diferem entre si pelo teste de Tukey $(\mathrm{p}<0,05)$.

** $\mathrm{n}=$ número de indivíduos avaliados

De acordo com os resultados obtidos no presente experimento, os indivíduos de $A$. colemani, das três regiões climáticas, apresentaram diferenças, tanto em suas características biológicas quanto na resposta as diferentes temperaturas, e algumas dessas respostas são provavelmente devido às adaptações a condições climáticas da área geográfica de origem. O número de indivíduos utilizados para iniciar as criações de A. colemani (dois casais) de cada uma das três regiões climáticas foi baixo para caracterizar as populações. Porém, foi observada existência de variação na resposta a temperatura, indicando que, apesar de A. colemani apresentar alta mortalidade em temperaturas elevadas, existem indivíduos com tolerância diferenciada, e que podem ser usados no controle biológico de $A$. gossypii em diferentes regiões e épocas do ano.

A temperatura mais adequada para o desenvolvimento do parasitóide $A$. colemani foi de $22^{\circ} \mathrm{C}$ para os indivíduos de Juramento e São Gotardo e de $25^{\circ} \mathrm{C}$ para aqueles de Lavras. Os indivíduos de $A$. colemani, de Juramento e São Gotardo, apresentaram aumento na mortalidade a 16 e $28^{\circ} \mathrm{C}$, enquanto que indivíduos de Lavras apresentaram alta sobrevivência em todas as temperaturas avaliadas. A alta emergência dos indivíduos de Lavras a $28^{\circ} \mathrm{C}$, demonstrou a existências de indivíduos de $A$. colemani com tolerância a temperaturas mais altas. Desta forma, novas perspectivas para a utilização de $A$. colemani no controle biológico de $A$. gossypii podem ser vislumbradas com a utilização de diferentes biótipos do parasitóide.

Agradecimentos. O primeiro e segundo autores agradecem ao $\mathrm{CNPq}$ pelas bolsas de estudo, e à FAPEMIG pelo suporte financeiro para a realização deste trabalho. Os autores agradecem também a Afonso C.V. Leme e Luis Carlos D. Rocha pela coleta dos parasitóides em São Gotardo e Juramento, respectivamente.

\section{REFERÊNCIAS}

Aspiazú, C.; G. A. Ribeiro; R. L. Vianello; J. C. Ribeiro; O. F. Valente \& F. Paula Neto. 1990. Análise dos componentes principais aplicados na classificação climática do Estado de Minas Gerais. Teste metodológico. Revista Árvore 14: 1-15.

Bartoszeck, A. B. 1976. Afídeos da ameixeira (Prunus domestica L.) e pessegueiro (Prunus persica Sto.), seus predadores e parasitas. Acta Biológica Paranaense 5: 69-90.

Blackman, R. L. \& V. P. Eastop 1984. Aphids on the world's crops: an identification guide. Chichester, J. Wiley, $466 \mathrm{p}$.

Bleicher, E. \& J. R. P. Parra. 1989. Espécies de Thrichogramma parasitóides de Alabama argillacea. I. Biologia de três populações. Pesquisa Agropecuária Brasileira 24: 929-940.

Bleicher, E. \& J. R. P. Parra. 1990a. Espécies de Thrichogramma parasitóides de Alabama argillacea. II. Tabela de fertilidade e parasitismo de três populações. Pesquisa Agropecuária Brasileira 25: 207-214.

Bleicher, E. \& J. R. P. Parra. 1990b. Espécies de Thrichogramma parasitóides de Alabama argillacea. III. Determinação das exigências térmicas de três populações. Pesquisa Agropecuária Brasileira 25: 215-219.

Botto, E. N.; D. González \& T. Bellows. 1988. Effect of temperature on some biological parameters of two populations of Aphidius ervi Haliday (Hymenoptera: Aphidiidae), p. 367-377. In: V. K. Grupta (Edit.). Advances in Parasitic Hymenoptera Research. Leiden and New York, Hardcover, $546 \mathrm{p}$.

Bueno, V. H. P. 1999. Protected cultivation and research on biological control of pests in greenhouses in Brazil. IOBC/WPRS Bulletin 22: 21-24

Bueno, V. H. P.; M. C. M. Soglia \& S. M. M. Rodrigues. 2004. Thermal requirement of Aphis gossypii Glover, 1877 (Hemiptera: Aphididae) when reared on different chrysanthemum cultivars, p. 345-349. In: J. C. Simon; C. A. Dedryver; C. Rispe \& M. Hullé (ed.). Aphids in a new millennium. Versailles, INRA-Editions, $549 \mathrm{p}$.

Hopper, K. R.; R. T. Roush \& W. Powell. 1993. Management of genetics of biological control introductions. Annual Review of Entomology 38: 27-51.

Liu, S.; F. B. Gebremeskel \& S. Shi. 2002. Reproductive compatibility and variation in survival and sex ratio between two geographical populations of Diadromus collaris, a pupal parasitoid of the 
diamondback moth, Plutella xylostella. Biocontrol 47: 625-643.

Messenger, P. S. \& R. Van Den Bosch. 1971. The adaptability of introduced biological control agents, p. 68-92. In: C. B. Huffaker (Edit.). Biological control. New York, Plenum Press, 511 p.

Ministério da Agricultura e Reforma Agrária, 1992. Normais climatológicas: 1961-1990. Brasília, 84 p.

Mols, P. J. M. \& J. M. Boers. 2001. Comparison of a Canadian and a Dutch strain of the parasitoid Aphelinus mali (Hald) (Hym., Aphelinidae) for control of woolly apple aphid Eriosoma lanigerum (Haussmann) (Hom., Aphididae) in the Netherlands: a simulation approach. Journal of Applied Entomology 125: 255-262.

Pimenta, H. R. \& J. G. Smith. 1976. Afídeos, seus danos e inimigos naturais em platações de trigo (Triticum sp.) no estado do Paraná. Curitiba, OCEPAR, $175 \mathrm{p}$.

Royer, T. A.; K. L. Giles; S. D. Kindler \& N. C. Elliott. 2001. Developmental response of three geographic isolates of Lysiphlebus testaceipes (Hymenoptera: Aphidiidae) to temperature. Environmental Entomology 30: 637-641.

Salvadori, J. R. \& L. A. B. Salles. 2002. Controle biológico dos pulgões do trigo, p. 427-448. In: J. R. P. Parra; P. S. M. Botelho; B. S. Corrêa-Ferreira \& J. M. S. Bento (eds.). Controle biológico no Brasil. São Paulo, Editora Manole, 635 p.

Sampaio, M. V. 2004. Bioecologia de Aphidius colemani Viereck, 1912 (Hymenoptera: Braconidae, Aphidiinae). Tese de Doutorado. Lavras, Universidade Federal de Lavras, $154 \mathrm{p}$.

Sampaio, M. V.; V. H. P. Bueno \& J. C. Van Lenteren. 2001. Preferência de Aphidius colemani Viereck (Hymenoptera: Aphidiidae) por Myzus persicae (Sulzer) e Aphis gossypii Glover (Hemiptera: Aphididae).
Neotropical Entomology 30: 655-660.

Silva, A. A. G. ; C. R. Gonçalves; D. M. Galvão; A. J. L. Gonçalves; J. Gomes; M. N. Silva \& L. Simoni. 1968. Quarto catálogo de insetos que vivem nas plantas do Brasil. Seus parasitos e predadores. Parte II, $3^{\circ}$ tomo. Rio de Janeiro, Ministério da Agricultura, xxvi+622 p.

Soglia, M. C. M.; V. H. P. Bueno \& M. V. Sampaio. 2002. Desenvolvimento e sobrevivência de Aphis gossypii Glover (Hemiptera: Aphididae) em diferentes temperaturas e cultivares comerciais de crisântemo. Neotropical Entomology 31: 211216.

Soglia M. C. M.; V. H. P. Bueno; S. M. M. Rodrigues \& M. V. Sampaio. 2003. Fecundidade e longevidade de Aphis gossypii Glover (Hemiptera, Aphididae) em diferentes temperaturas e cultivares comerciais de crisântemo (Dendranthema grandiflra Tzvelev). Revista Brasileira de Entomologia 47: 49-54.

Toussidou, M.; M. C. Williams \& S. Leather. 1999. Life history parameters of Aphidius colemani (Hym.: Aphidiidae) on sweet pepper in different temperature regimes. IOBC/WPRS Bulletin 22: $255-258$

Van Steenis, M. J. 1993. Suitability of Aphis gossypii Glov., Macrosiphum euphorbiae (Thom.) and Myzus persicae Sulz. (Hom.: Aphididae) as host for several aphid parasitoid species (Hym.: Braconidae). IOBC/WPRS Bulletin 16: 157-160.

Van Steenis, M. J. \& K. A. M. H. El-Kawass. 1995. Life history of Aphis gossypii on cucumber: influence of temperature, host plant, and parasitism. Entomologia Experimentalis et Applicata 76: $121-131$. 